\title{
Activation of septal OXTr neurons induces anxiety- but not depressive-like behaviors
}

\author{
Tuanjie Huang ${ }^{1,2}$, Fangxia Guan ${ }^{2}$, Julio Licinio $\mathbb{D D}^{3}$, Ma-Li Wong $\mathbb{D}^{3}$ and Yunlei Yang $\mathbb{D}^{1,4,5,6 \times}$ \\ (c) The Author(s) 2021
}

The neuropeptide oxytocin (OXT) is well recognized for eliciting anxiolytic effects and promoting social reward. However, emerging evidence shows that OXT increases aversive events. These seemingly inconsistent results may be attributable to the broad OXT receptor (OXTr) expression in the central nervous system. This study selectively activated septal neurons expressing OXTr using chemogenetics. We found that chemogenetic activation of septal OXTr neurons induced anxiety- but not depressive-like behavior. In addition, septal OXTr neurons projected dense fibers to the horizontal diagonal band of Broca (HDB), and selective stimulation of those HDB projections also elicited anxiety-like behaviors. We also found that septal OXTr neurons express the vesicular GABA transporter (vGAT) protein and optogenetic stimulation of septal OXTr projections to the HDB inactivated HDB neurons. Our data collectively reveal that septal OXTr neurons increase anxiety by projecting inhibitory GABAergic inputs to the HDB.

Molecular Psychiatry (2021) 26:7270-7279; https://doi.org/10.1038/s41380-021-01283-y

\section{INTRODUCTION}

It has been well-demonstrated that anxiety is under the control of multiple neuronal populations and complex neural circuits primarily concentrated in the limbic system. Previous studies with local OXTr (oxytocin receptor) mRNA assays $[1,2]$ or OXTr reporter mice [3-5] have demonstrated that OXTr is widely expressed in mammalian brains, including the septum, nucleus accumbens, and ventral tegmental area [6, 7]. Previous studies have shown that the genes encoding the neuropeptide OXT are predominantly expressed in the magnocellular neurons of the paraventricular nucleus of the hypothalamus (PVN) and supraoptic nuclei (SON) [8]. In addition to the peripheral release of the processed OXT peptide from the posterior pituitary in response to various stimuli, oxytocinergic neurons in the PVN project to many different brain regions in the central nervous system (CNS) [9].

Previous studies have been focused on the functional roles of OXT in social reward-associated brain regions, including the nucleus accumbens [10] and ventral tegmental area [11,12]. There is ample evidence showing that OXT elicits anxiolytic effects [13-17] and ameliorates depression [18], while emerging evidence indicates that OXT also exerts anxiogenic effects [19]. These seemingly inconsistent findings could be reconciled because the OXTr is widely expressed in the CNS, and different experimental paradigms were used in those studies. Neuronal populations participating in the anxiogenic effects of OXT remain to be identified.

Recently we showed that selective stimulation of glutamatergic inputs of the ventral hippocampus (VHPC) in the septum and septal vGAT (Vesicular GABA transporter) and vGluT2 (Vesicular glutamate transporter 2) neurons suppressed food intake [20-22], which suggests that the VHPC modulates hypothalamic feeding centers via the septum. We have also shown that feeding may change anxiety levels [23].

We observed that septal OXTr neurons expressed vGAT; however, their roles in regulating anxiety remain unclear. We have performed a series of experiments to define septal OXTr neurons' roles in regulating anxiety- and depressive-like behavior and deciphered their downstream target. We employed a chemogenetic DREADD (Designer Receptor Exclusively Activated by Designer Drug) approach to manipulate cell-type-specific neuronal populations in a spatiotemporal manner. Our results show that DREADD stimulation of septal OXTr neurons or their projections in the HDB (Horizontal diagonal band of Broca) increases anxiety- but not depressive-like behaviors. We also found that activation of septal OXTr neuron projections to the HDB inhibits HDB neurons. Together, previous literature and our results in this study let us posit that septal neurons modulate anxiety- and depressive-like behaviors by projecting to different downstream targets.

\section{METHODS}

The Albert Einstein College of Medicine's Institutional Animal Care and Use Committees approved all experimental protocols, which were conducted according to the US National Institutes of Health guidelines for animal research.

\section{Animals}

We used both male and female C57BL/6 J and Oxtr-Cre (Jax 031303, Jackson Lab, Bar Harbor, ME) mice (age 5-8 weeks) at the start of experiments. We group-housed $3-5$ mice per cage in temperature- $\left(22-25^{\circ} \mathrm{C}\right)$ and

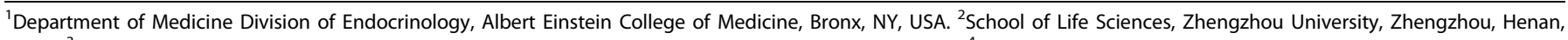

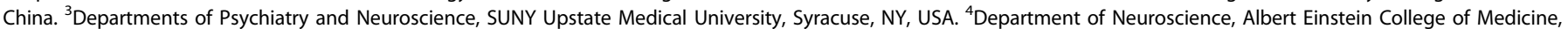

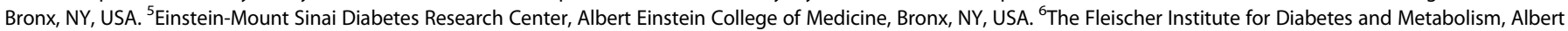
Einstein College of Medicine, Bronx, NY, USA. ${ }^{凶}$ email: yunlei.yang@einsteinmed.org 
humidity-controlled rooms on a 12-h light:12-h dark cycle, with lights on from 8:00 am to $8: 00 \mathrm{pm}$ and with ad libitum access to mouse standard chow (PicoLab Rodent Diet 20, 5058, LabDiet, St. Loius, MO) and water. Mice were single-caged after they received viral transductions with or without guide cannula or fiber insertions until we finished all the experimental procedures. The virally transduced mice were randomly and evenly matched for age and sex.

\section{Pharmacology}

All chemicals were purchased from Sigma (Millipore Sigma, St Louis, MO) except for JHU37160 (J60; water-soluble) which was purchased from HelloBio Inc (Princeton, NJ). We performed intraperitoneal (i.p.) injections using 27-gauge needles and diluted the stocks of $J 60$ in saline $(200 \mu \mathrm{l})$ at the final dose of $J 60(1 \mathrm{mg} / \mathrm{kg})$ on the experimental days. For experiments requiring intra-HDB administration, an injector (33 GA, Plastics One) with a $1-\mathrm{mm}$ extension beyond the guide cannula $(3.5 \mathrm{~mm}$ length; $26 \mathrm{GA}$, Plastics One) was attached to a Hamilton syringe via polyethylene tubing. We used a micromanipulator (Narishige International Inc, Amityville, NY) to control the injection speed at $50 \mathrm{nl}$ per min for $2 \mathrm{~min}$ and withdrawn the injector $2 \mathrm{~min}$ after the final injection. The guide cannula was inserted into the brain for intra-HDB injections and anchored to the skull using grip cement (Dentsply Sirona, Charlotte, NC). A dummy cannula (33 GA, Plastics One, Torrington, CT) was used to prevent cannula clogging. The amount for intra-HDB was $100 \mathrm{nl}$ of $J 60$ $(1 \mathrm{mg} / \mathrm{kg})$.

\section{Viral Vectors}

Viral vectors used in this study included: AAV vectors for the Cre-dependent hM3Dq ( $\mathrm{AAV}_{2}$-hSyn-DIO-hM3Dq-mCherry, addgene\#44361, titer at $7.8 \times$ $\left.10^{12} \mathrm{vg} / \mathrm{ml}\right), \mathrm{hM} 4 \mathrm{Di}\left(\mathrm{AAV}_{2}\right.$-hSyn-DIO-hM4Di-mCherry, addgene\#44362, titer at $1.27 \times 10^{13} \mathrm{vg} / \mathrm{ml}$ ), or ChR2 [(AAV5-EF1a-DIO-hChR2(H134R)-EYFP-WPREHGHpA), addgene\#20298, titer at $\left.1.0 \times 10^{13} \mathrm{vg} / \mathrm{ml}\right]$. AAV vectors for non-Cre dependent GCaMP ${ }_{6 s}\left(A_{A} V_{1}\right.$-hSyn1-GCaMP6s-P2A-nls-dTomato, addgene\#51 084 , titer at $5.0 \times 10^{12} \mathrm{vg} / \mathrm{ml}^{\prime} \mathrm{AAV}_{1}$-hSyn1-axon-GCaMP6s, addgene\#111262, titer at $1.0 \times 10^{13} \mathrm{vg} / \mathrm{ml}$ ). Control vectors were used (AAV-hSyn-DIO-mCherry, addgene\#50459, titer at $1.5 \times 10^{13} \mathrm{vg} / \mathrm{ml}$; AAV-hSyn-EGFP, addgene\#50465, titer at $8.0 \times 10^{12} \mathrm{vg} / \mathrm{ml}$ ). Aliquoted viral vectors were stored at $-80^{\circ} \mathrm{C}$ until used for stereotaxic injections.

\section{Stereotaxic viral injections and cannula implantation for intra- HDB injections}

Surgical procedures have been detailed in our recent publications $[20,21,24]$. Briefly, mice were anesthetized with isoflurane $(1.5-3.0 \%)$ and placed in a stereotaxic frame (Harvard Apparatus, Holliston, MA). The mouse skull was exposed via a small incision, and two small holes were drilled directly above the viral injection sites bilaterally using a microprecision drill (CellPoint Scientific, Gaithersburg, MD). A glass pipette with $20-40 \mu \mathrm{m}$ tip diameter was inserted into each side of the brain, and two injections ( $300 \mathrm{nl}$ each side) of the vectors were delivered into the lateral septum at coordinates (bregma $+0.5 \mathrm{~mm}$; midline $\pm 0.4 \mathrm{~mm}$; dorsal surface $-2.2 \mathrm{~mm}$ ), or into the vHPC at coordinates (bregma $-3.2 \mathrm{~mm}$; midline $+3.2 \mathrm{~mm}$; dorsal surface $-3.8,-3.5,-3.2 \mathrm{~mm}$ ). A micromanipulator (Narishige) was used to control the viral injection at a speed of $30 \mathrm{nl}$ per min, and the injection pipette was withdrawn $15 \mathrm{~min}$ after the final injection to assure adequate viral delivery. For the experiments using intra-HDB injections, a customer-made bilateral 26gauge stainless steel cannula ( $3.5 \mathrm{~mm}$ length, Plastics One) was inserted through the craniotomy. For photometry experiments, a TeleFipho fiberoptic cannula (fiber core at $400 \mu \mathrm{m} / \mathrm{NA} \mathrm{0.39}$, cladding at $425 \mu \mathrm{m}$, ferrule diameter at $2.5 \mathrm{~mm}$; Amuza $\mathrm{Inc}$ ) was implanted over the $\mathrm{VHPC}$ (coordinate at bregma $-3.2 \mathrm{~mm}$; midline $+3.2 \mathrm{~mm}$; dorsal surface $-3.0 \mathrm{~mm}$ ) or over the lateral septum (coordinate at bregma $+0.5 \mathrm{~mm}$; midline $+0.4 \mathrm{~mm}$; dorsal surface $-2.0 \mathrm{~mm}$ ). The guide cannula or fiberoptic cannula was anchored to the skull with grip cement (Dentsply). Stainless dummies inserted into the guide cannula prevented the guide cannula from clogging. Optic fiber dust caps were placed on optic fibers to keep the fibers clean. Mice were returned to their home cages and singly housed typically for at least two weeks for recovery and viral expression before performing the experiments.

\section{Open field test (OFT)}

OFTs were performed following the procedures detailed in our previous studies $[23,25,26]$. In brief, virally transduced mice were habituated to the behavioral room for $2 \mathrm{~h}$ before beginning the experimental sessions. The open field consisted of a brightly lit $40 \times 40 \mathrm{~cm}$ arena. We defined the center of the arena at $20 \times 20 \mathrm{~cm}$ area. Mice received i.p. $J 60(1 \mathrm{mg} / \mathrm{kg})$ 30 min before the 10-min test using ANY-maze software (Stoelting Co. Wood Dale, IL). Between the trials, we cleaned the arena with $70 \%$ ethanol.

\section{Elevated-plus maze (EPM)}

Similar to the OFT, virally transduced mice were habituated to the behavioral room for $2 \mathrm{~h}$. We placed the animals in the center of the start zone and allowed them to explore the arms for $5 \mathrm{~min}, 30 \mathrm{~min}$ after receiving i.p. J60 (1 mg/kg). ANY-Maze software (Stoelting Co.) monitored and recorded the animals' exploration. The arena was cleaned with $70 \%$ ethanol between trials.

\section{Sucrose preference test (SPT)}

We followed the procedure as described in previous studies [27] with modifications. Briefly, we adapted the mice in their home cages for $72 \mathrm{~h}$ to two liquid diet feeding tubes $(50 \mathrm{ml}$; Bio-SerV, \#9019, Flemington, NJ) containing $1 \%$ sucrose in water ( $\mathrm{wt} / \mathrm{vol})$ and tap water, respectively. We changed the positions of the two tubes every $24 \mathrm{~h}$. Mice had free access to lab chow. Each animal was given one sucrose tube and one water tube for $14 \mathrm{~h}(7: 00 \mathrm{pm}$ to $9: 00 \mathrm{am})$ during the baseline measurement. During the preference test on the following day, mice were deprived of both food and water for $12 \mathrm{~h}(7: 00 \mathrm{am}$ to 7:00 pm). After which and $30 \mathrm{~min}$ before the test mice were treated with $\mathrm{J} 60$ or vehicle via i.p. injections and provided with one sucrose tube and one water tube for $14 \mathrm{~h}(7: 00 \mathrm{pm}$ to 9:00 am). The sucrose solution and water weights were recorded, and the percent sucrose preference index was calculated using the following formula: Sucrose preference index $=$ [sucrose consumption/ (water + sucrose consumption)] $\times 100 \%$.

\section{Social behavioral test}

Thirty min after i.p. $J 60$ or vehicle treatment, two age- and sex-matched mice were placed in one chamber $(20 \times 30 \mathrm{~cm})$. As described in previous studies [28, 29], exploratory behaviors were recorded for $20 \mathrm{~min}$ and analyzed using a social behavior software (Video Capturing and TopScan High-Throughput Option Version 3.0, CleverSys Inc., Reston, VA). Between the trials, the arena was cleaned with $70 \%$ ethanol. Active contact, passive contact, social approach, social leave, social follow, and social sniff were recorded. Bouts of social interaction and social interaction duration were calculated for every group using the formula: social interaction $=$ social follow + social sniff.

\section{Electrophysiology and optogenetic stimulation}

Following our recently published protocols $[20,22,24]$, acute coronal sections that included the septum and/or HDB were prepared from the septal OXTr neuron ChR2-, hM3Dq-, hM4Di-, or control fluorescent protein-transduced Oxtr-Cre mice. Briefly, mice were deeply anesthetized with isoflurane and decapitated. Mouse brains were dissected rapidly and placed in ice-cold oxygenated $\left(95 \% \mathrm{O}_{2}\right.$ and $\left.5 \% \mathrm{CO}_{2}\right)$ solution containing (in mM): 110 Choline Chloride, $2.5 \mathrm{KCl}, 1.25 \mathrm{NaH}_{2} \mathrm{PO}_{4}, 2 \mathrm{CaCl}_{2}$, $7 \mathrm{MgSO}_{4}, 25 \mathrm{D}$-glucose, $3.1 \mathrm{Na}$-pyruvate and $11.6 \mathrm{Na}$-L-ascorbate, pH 7.3. Coronal brain slices (260- $\mu \mathrm{m}$ thickness) were cut with a vibratome (Leica; VT $1200 \mathrm{~S}$ ) and maintained in an incubation chamber at $34{ }^{\circ} \mathrm{C}$ for $30 \mathrm{~min}$ and then brought to room temperature until being transferred to a recording chamber. During experiments, an individual slice was transferred to a submersion-recording chamber and continuously perfused with recording solution containing the following (in $\mathrm{mM}$ ) $119 \mathrm{NaCl}, 25 \mathrm{NaHCO}_{3}, 11 \mathrm{D}$-glucose, $2.5 \mathrm{KCl}, 1.25 \mathrm{MgCl}_{2}, 2 \mathrm{CaCl}_{2}$, and 1.25 $\mathrm{NaH}_{2} \mathrm{PO}_{4}$, aerated with $95 \% \mathrm{O}_{2} / 5 \% \mathrm{CO}_{2}\left(1-2 \mathrm{ml}\right.$ per min at $\left.30^{\circ} \mathrm{C}\right)$. Virally transduced neurons or their projections were identified using an Olympus microscope (BX51WI) and observed by fluorescence emission. Loose-seal cell-attached recordings were made through the experiments using an Axo-patch 700 B amplifier (pClamp 11.0 software, Molecular Devices, San Jose, CA). The recording electrodes had tip resistances of 3-5 $\mathrm{M} \Omega$ and seal resistances of 20-70 $\mathrm{M} \Omega$. Recording pipettes were filled with artificial cerebrospinal fluid (aCSF). Recordings were initiated $5 \mathrm{~min}$ after the loose-seal cell-attached recordings were established. For the DREADD experiments, J60 was added to the circulating aCSF after 2-5 min of baseline recordings. For the ChR2 experiments, blue lights were delivered on the surface of brain slices using an optic fiber connected to a blue laser power (Crystal Laser $473 \mathrm{~nm}$ ), and light pulse ( $3 \mathrm{~ms}$ duration) was controlled by the pClampfit software. Responses 
were digitized at $10 \mathrm{kHz}$ through whole experiments using Multi-Clamp 700B amplifier and analyzed with pClampfit 11 software (Molecular Devices). For detection of action potentials, we used template matching (pClampfit) followed by visual inspection.

\section{Wireless fiber photometry (TeleFipho) in freely moving mice} For experiments performing TeleFipho recordings, vHPC neurons or their axons were transduced with $\mathrm{GCaMP}_{65}$. A unilateral TeleFipho fiber-optic cannula ( $400 \mu \mathrm{m}$ core, 0.39 numeric aperture, $425 \mu \mathrm{m}$ cladding, and 2.5 $\mathrm{mm}$ diameter; Amuza Inc) was implanted over the vHPC or the lateral septum. A TeleFipho system (Amuza Inc), including the small and lightweighted headstage and wireless transmission circuit, was set and adjusted with technical help. The headstage ( $3 \mathrm{~g}$ weight; $12 \times 12 \mathrm{x}$ $22 \mathrm{~mm}$ dimensions) is composed of a filter cub, photodetector, and blue light source (excitation wavelength peaked at $470 \mathrm{~nm}$ with $445-490 \mathrm{~nm}$ filter band; emission wavelength with a $500-550 \mathrm{~nm}$ band). The sampling rate was set at $100 \mathrm{~Hz}$ with a gain at $10^{10} \mathrm{~V} / \mathrm{A}$. The signal transmission distance was within $2 \mathrm{~m}$. The receiver containing a $1 \mathrm{x}$ photometry analog out and $1 \mathrm{x}$ general-purpose analog were connected to a PC installed with the TeleFipho software. The light power was adjusted to reach a baseline of around 35000 following the software instructions to achieve maximum sensitivity and avoid saturating the detector. The maximum power of the $465 \mathrm{~nm}$ was $300 \mu \mathrm{W}$. We normalized the $\mathrm{GCaMP}_{6 \mathrm{~s}}$ signal intensity (I) for each trial and calculated the $Z$ score as $\left(I-I_{\text {mean }}\right) / I_{S D}$, where $I_{\text {mean }}$ and $I_{S D}$ represent the mean and standard deviation of the signals for each mouse. The TeleFipho experiments were performed $5 \mathrm{~min}$ after connecting the optic fibers to the animals and before placing the mice in the open arms of the EPM or the light chamber of a light-dark box (LDB), respectively, for 5- or 10-min free explorations. The LDB consists of a box $(60 \mathrm{~cm} \times 40 \mathrm{~cm} \times 20 \mathrm{~cm})$ divided into a small $(20 \mathrm{~cm} \times 40 \mathrm{~cm} \times 20 \mathrm{~cm})$ dark chamber and a large $(40 \mathrm{~cm} \times 40 \mathrm{~cm} \times 20 \mathrm{~cm})$ brightly lit chamber. A small opening $(5 \mathrm{~cm}$ high by $4 \mathrm{~cm}$ wide) located at floor level in the center of the dividing plate connects the two chambers.

\section{Immunofluorescence assays}

Mice were perfused with $0.1 \mathrm{M}$ phosphate-buffered saline (PBS) in $\mathrm{pH}$ 7.4, followed by $4 \%$ paraformaldhyde (PFA) in PBS and then $0.1 \mathrm{M}$ PBS. Septal coronal brain sections $(40 \mu \mathrm{m})$ were obtained using a vibratome (Leica VT1200, Leica Biosystems, Buffalo Grove, IL) and mounted on glass slides. The virally transduced mice received i.p. injections of $J 6030$ min before the perfusion. Briefly, brain sections were washed three times in washing buffer $(0.1 \%$ Triton X-100 in $0.1 \mathrm{M} \mathrm{PBS})$ for $10 \mathrm{~min}$, followed by permeabilization in a solution containing $1 \%$ Triton $\mathrm{X}-100$ in $0.1 \mathrm{M}$ PBS) for $30 \mathrm{~min}$ at room temperature, and then blocked for $2 \mathrm{~h}$ in a blocking solution ( $5 \%$ bovine serum in washing buffer). Brain sections were then incubated overnight at $4{ }^{\circ} \mathrm{C}$ with primary antibodies diluted in PBS, supplemented with $1 \%$ BSA and $0.1 \%$ Triton X-100, and washed three times and incubated overnight at $4{ }^{\circ} \mathrm{C}$ with Alexa fluoro 647-conjugated anti-Fos antibody (E-8) (1:150; sc-166940; Lot\#A3020; Santa Cruz Biotechnology Inc., Dallas, TX); and the vGAT was stained using vGAT Polyclonal antibody (1:25, Invitrogen, Thermo Fisher Scientific, Waltham, MA) respectively. Sections were washed three times using washing buffer for $2 \mathrm{~h}$ at room temperature, incubated with the goat anti-rabbit IG-conjugated with Alexa fluoro 594 (1:1,000; Invitrogen) for vGAT-stain at room temperature for $2 \mathrm{~h}$, and then washed three times for $10 \mathrm{~min}$ each time using washing buffer and mounted on glass slides with Fluoromount-G mounting medium (Southern Biotech, Birmigham, AL). Images ( $2 \mu \mathrm{m}$ thickness, five images) were collected using 20X and $40 \mathrm{X}$ objectives in a confocal microscope (Leica SP8).

\section{Statistics and reproducibility}

Animals were randomly assigned to experimental (DREADD) or control groups (control fluorescent protein transduced) before viral injections and behavioral experiments. Only mice with accurate viral injections and cannula placements were included in the data analysis. All experiments were repeated twice for each mouse, and average values were calculated for each mouse for statistical analysis. Differences between two groups were analyzed using Student's $t$-tests or Mann-Whitney tests when appropriate. Comparison of more than two groups was made using oneway ANOVA with Tukey's post hoc test. Repeated measures (RM) two-way ANOVA with the within-subject factors of time segment and treatment (vehicle vs. J60) or mixed ANOVA with the within-subject factor of time segment and the between-subjects factor of viral injections type was used to analyze data from more than two groups across various time points. Sidak's post hoc test was used to test from significant effects at various time segments following the detection of a significant main effect or interaction. Homoscedasticity and normality parameters were obtained in male and female data. All data were analyzed using Prism 8.0 (GraphPad Software).

\section{RESULTS}

DREADD activation of OXTr expressing neurons in the septum We first validated septal OXTr neuron activation with a chemogenetic DREADD approach, which was employed to selectively stimulate or inhibit these neurons in Oxtr-Cre transgenic mice. Following our previously published protocols $[20,21,24]$, we targeted vectors respectively carrying Credependent stimulatory hM3Dq, inhibitory hM4Di, or control mCherry to the lateral septum (LS) in Oxtr-Cre mice (Fig.1a), and the proteins were predominantly expressed in the septum and OXTr neuron projections to downstream targets including the HDB (Fig. 1a). We applied the in vivo DREADD agonist JHU37160 dihydrochloride (J60), which has been verified in our recent studies [24], to evaluate the ability of J60 to regulate OXTr neuron activity. We observed that i.p. injection of $J 60 \quad(1 \mathrm{mg} / \mathrm{kg})$ significantly increased Fos-positive OXTr neurons in the hM3Dqtransduced mice compared to the mCherry-transduced ones (Fig. 1 b, c).

Moreover, we performed in vitro loose-seal cell-attached recordings from septal OXTr neurons in acute brain slices in the virally transduced Oxtr-Cre mice. J60 $(10 \mu \mathrm{M})$ was added to the circulating aCSF after a stable baseline recording. Action potential firing rates were analyzed and compared before and after the application of J60. Consistent with the above Fos results, we observed that $\mathrm{J} 60$ potently increased the action potential firing rates in the hM3Dq-transduced OXTr neurons compared to mCherry-transduced neurons (Fig. 1d-f). In addition, J60 decreased the action potential firing rates of hM4Di-transduced OXTr neurons (Fig. 1f). These results indicate that the DREADD approach with $\mathrm{J} 60$ treatment can be reliably utilized to correlate neuron activities to animal behaviors.

\section{DREADD activation of septal OXTr neurons induces anxiety} We performed a series of animal behavioral tests to evaluate the septal OXTr neurons' capability to regulate anxiety- and depressive-like behaviors by activating or inactivating septal OXTr neurons of hM3Dq-, hM4Di-, or mCherry-transduced mice respectively. The virally transduced mice received $\mathrm{J} 60$ treatment via i.p. injections $30 \mathrm{~min}$ before the behavioral tests. We observed that DREADD activation of septal OXTr neurons induced anxietylike behaviors in both males and females, as indicated by the decreased time spent and number of entries in the center of the open field (Fig. 2a-d), and decreased percent center distance (Fig. 2e) in activated hM3Dq-transduced mice compared to the mCherry-transduced mice. We also noticed that septal OXTr neuron activation in male mice significantly decreased total distance in the OFT (Fig. 2f).

To further confirm the anxiogenic effects of septal OXTr neurons, we performed the EPM test. Consistent with the OFT findings, we observed that activation of the septal OXTr neurons with $\mathrm{J} 60$ via i.p. injections diminished the time spent (Fig. $2 \mathrm{~g}-\mathrm{i}$ ) and the number of entries in the EPM's open arms (Fig. 2j) in hM3Dq-transduced mice compared to mCherry-transduced animals, and hM3Dq-transduced female mice had a small but significant decreased in the total distance traveled (Fig. 2k). DREADD inactivation of septal OXTr neurons with i.p. injection of J60 did not exert effects in hM4Di-transduced mice compared to mCherry-transduced mice, as indicated by no differences in the 
a

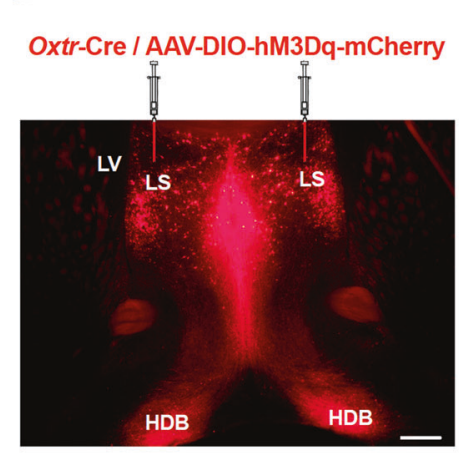

b

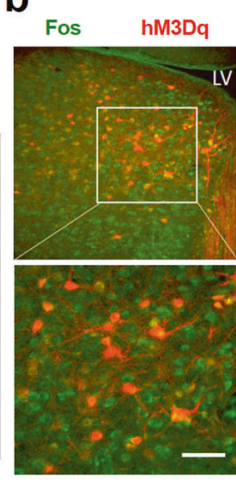

C

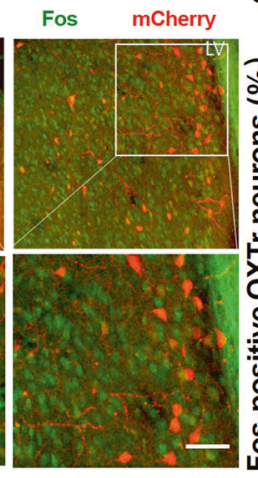

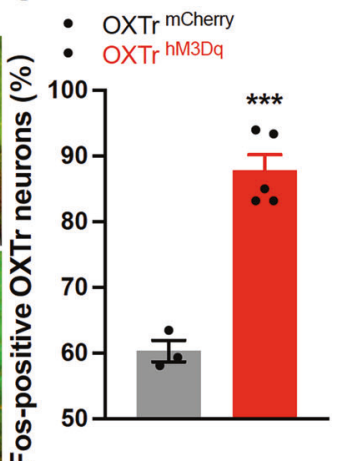

d
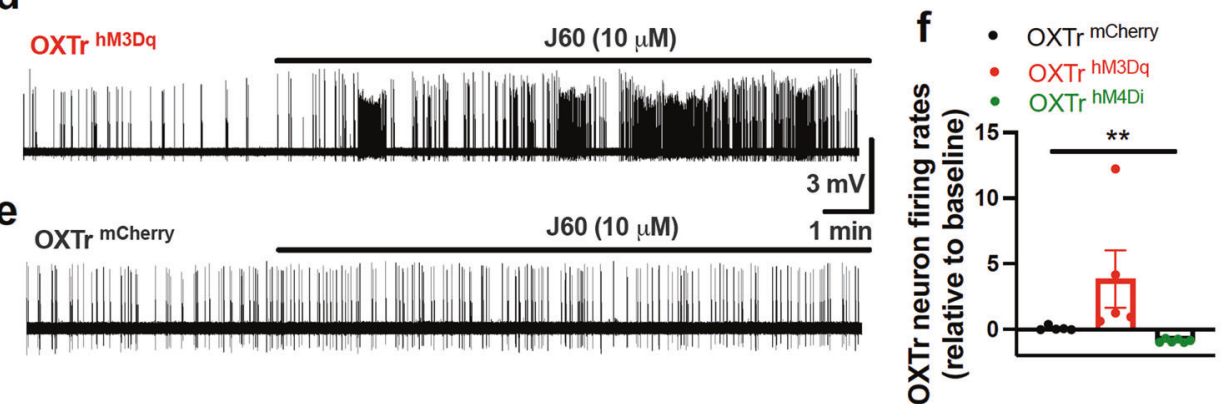

Fig. 1 DREADD activation of septal OXTr neurons. a A representative image of hM3Dq-mCherry-transduced OXTr neurons in the septum and their projections to the HDB. b, c J60 treatment via i.p. injections potently increased Fos positive OXTr neurons in the hM3Dq-mCherry transduced mice $(n=5)$ compared to mCherry-transduced mice $(n=3)$ : (b) Representative images of Fos and hM3Dq-mCherry in the septal OXTr neurons of hM3Dq-mCherry transduced animals; (c) Quantification of the percentage of Fos positive OXTr neurons transduced with hM3Dq-mCherry (hM3Dq, $n=5$; mCherry; $n=3 ; p=0.0002$; Unpaired student $t$ test). d-f Loose-seal cell-attached recordings: adding J60 to the circulating aCSF increased the firing rates at the (d) hM3Dq-transduced OXTr neurons but not the (e) mCherry-transduced neurons in acute brain slices of the virally transduced Oxtr-Cre mice; (f) Group data of action potential firing rates relative to baseline in hM3Dq- hM4Di-, or mCherry-transduced OXTr neurons with $J 60$ ( $n=5$ per group). One-way ANOVA was used for (f), and an unpaired student $t$-test for (c). Mean \pm SEM; ${ }^{* *} p<0.01 ;{ }^{* * *} p<0.001$. Scale bars, $2 \mathrm{~mm}$ for (a); and $50 \mu \mathrm{m}$ for (b).

time spent in the center, center distance, and the number of crossing in the center of the open field (Fig. 3a-c), and no differences in the time spent in open arms and the number of entering the open arms (Fig. 3d, e) of the EPM.

\section{Activation of septal OXTr neuronal projections to HDB induces anxiety-related behaviors}

We next tried to identify the circuitry underlying the anxiogenic effects of septal OXTR neurons. As stated above, we observed that septal OXTr neurons project dense fibers to the HDB (Fig. 1a). To determine the behavioral relevance of septal OXTr neural projections to the HDB, we transduced septal OXTr neurons with the stimulatory $\mathrm{hM} 3 \mathrm{Dq}$ by injecting a vector carrying Credependent $\mathrm{hM} 3 \mathrm{Dq}$ in the lateral septum of Oxtr-Cre mice and implanting a guide cannula to perform intra-HDB injections (Fig. 4a). We observed that activating septal OXTr neural projections with intra-HDB $\mathrm{J} 60$ injections induced anxiety, as indicated by the decreased time spent in the center (Fig. $4 b-c$ ), percent distance traveled in the center (Fig. 4d), and number of center entries (Fig. 4e) of the open field in the OXTr neuron hM3Dq-transduced mice compared to the OXTr neuron mCherrytransduced mice. To further confirm these projections' anxiogenic effects, we performed the EPM test (Fig. 4f-h). Matching with the above OFT results, we observed that stimulation of OXTr neural projections by $\mathrm{J} 60$ via intra-HDB injection decreased the time spent in the open arms (Fig. 4f, g) and open arm entering number (Fig. 4h) in the OXTr neuron hM3Dq-transduced mice compared to the mCherry-transduced mice.

\section{Septal OXTr neurons do not modulate social and sucrose preference behaviors}

To evaluate whether septal OXTr neurons modulate animal sociability, we performed social behavioral tests by placing one pair of virally transduced age- and gender-matched mice in one open chamber for $20 \mathrm{~min}$, following published protocols [28, 29]. Time engaged in social interactions, including the social following and head-body-genital sniffing for the paired mice (combining behaviors for both mice), were recorded and analyzed using TopScan software (CleverSys Inc.). We observed that chemogenetic activation of septal OXTr neurons with $\mathrm{J} 60$ via i.p. injections did not elicit effects on social behaviors, as there were no differences in the bouts (Fig. 5a) and duration (Fig. 5b) of social interactions between the septal OXTr neuron hM3Dq-, hM4Di-, and mCherry-transduced mice. We also performed the sucrose preference test (SPT) in the transduced animals and noticed that i. p. injection of $\mathrm{J} 60$ did not cause alterations in sucrose preference among them (Fig. $5 \mathrm{c}$ ). These results indicate that septal OXTr neurons do not modulate sociability and sucrose preference, suggesting that these neurons do not regulate depressive-like behaviors.

\section{Optogenetic stimulation of septal OXTr projections to the HDB inhibits HDB neurons}

Previous studies demonstrate that the lateral septum is mainly composed of GABAergic neurons [30,31], and the vGAT is a neuronal marker of GABAergic neurons in the central nervous system $[32,33]$. We thus posited that septal OXTr neurons are a 


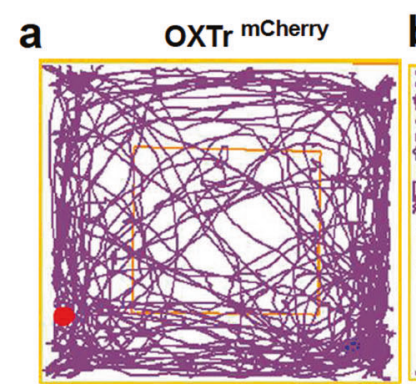

C

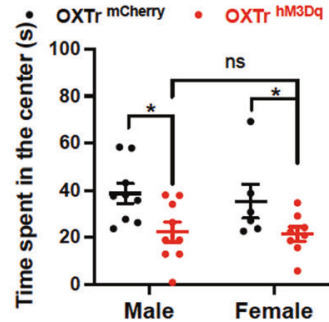

e

- OXTr mcherry - OXTr hM3Dq

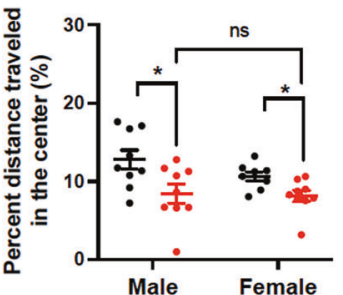

b OXTr hM3Dq

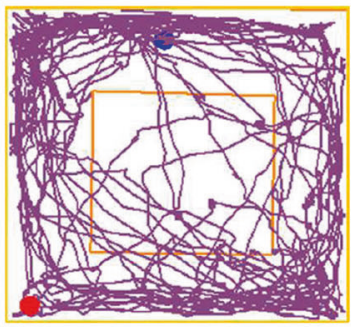

d

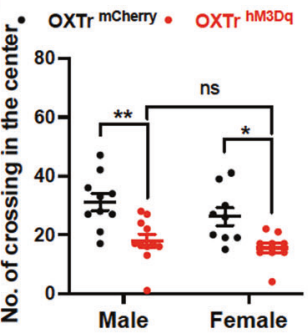

f

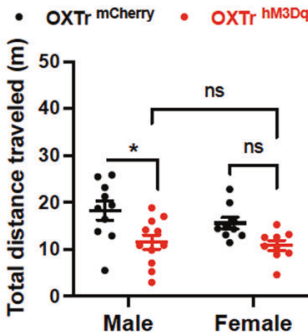

g

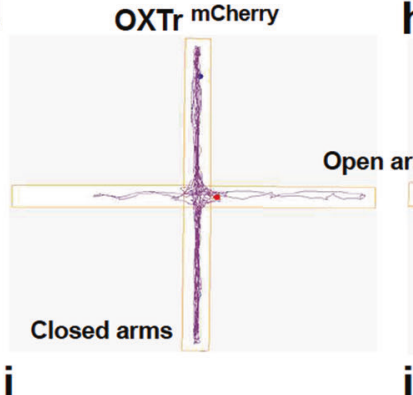

क. OXTr mCherry - OXTr hM3Dq

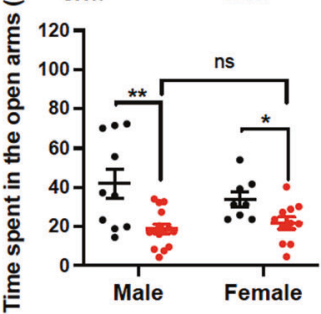

j

Closed arms

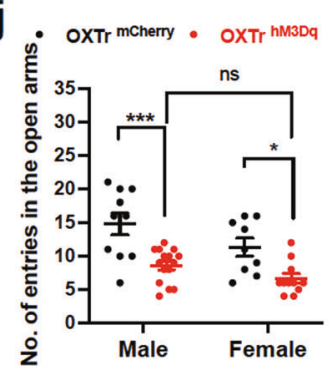

k

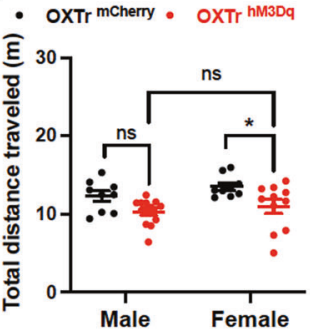

Fig. 2 Activation of septal OXTr neurons induced anxiety in both males and females. a, b Representative open field test (OFT) tracks of the septal OXTr neuron of (a) mCherry- or (b) hM3Dq-transduced Oxtr-Cre mice. c Time spent in center of the open field (male mCherry, $n=9$; male $\mathrm{hM} 3 \mathrm{Dq}, n=9$; female mCherry, $n=6$; female $\mathrm{hM} 3 \mathrm{Dq}, n=8$ ). $\mathbf{d}$ The number of entries in the center of the open field. e Percent distance traveled in the center. $\mathbf{f}$ Total traveled distance during the OFT. $\mathbf{g}, \mathbf{h}$ Representative elevated plus-maze (EPM) tracks of the septal OXTr neuron of $(\mathbf{g})$ mCherry- or (h) hM3Dq-transduced mice (male mCherry, $n=10$; male hM3Dq, $n=15$; female mCherry, $n=9$; female hM3Dq, $n=11$. $\mathbf{i}$ Time spent in the open arms of the EPM. $\mathbf{j}$ The number of entries in the open arms. $\mathbf{k}$ Total distance traveled in the EPM. Mice were treated with $J 60$ i.p. 30 min before the OFT and EPM tests. Two-way ANOVA was used to analyze all panels. Student t-tests were used for female data (c, i). Mean \pm SEM; ${ }^{*} p<0.05 ;{ }^{* *} p<0.01 ;{ }^{* * *} p<0.001$; ns not significant.
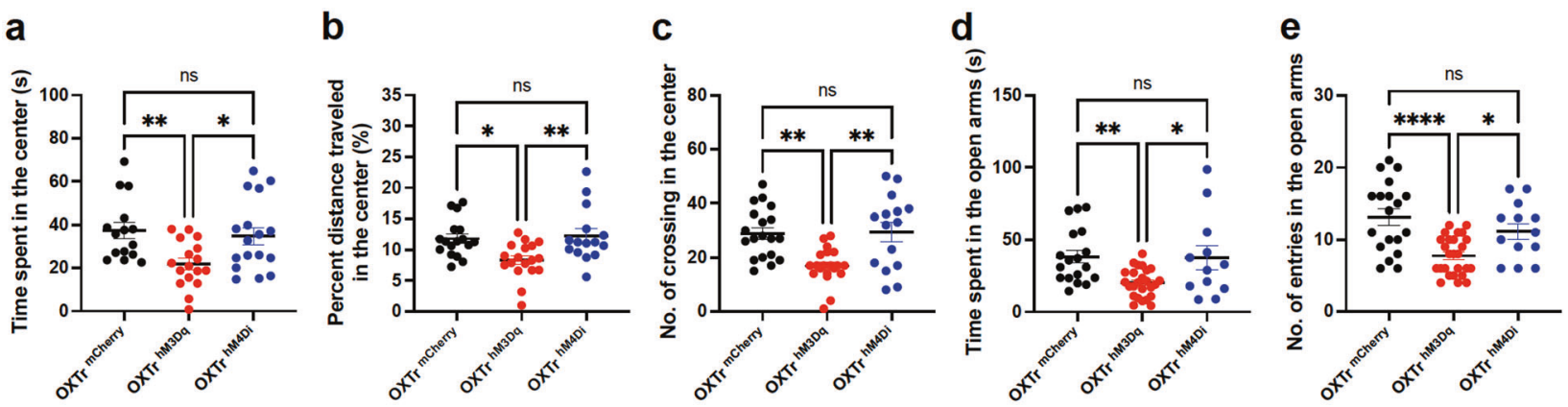

Fig. 3 Inactivation of septal OXTr neurons did not elicit anxiety behavior. a-c Open-field behavioral tests (OFTs) were performed in the septal OXTr neuron in hM3D $(n=23)-$, hM4Di $(n=17)$-, or mCherry $(n=20)$-transduced mice: (a) Time spent in the center of open field; (b) Percent distance traveled in the center; and (c) The number of entries in the center of the open field. d, e Elevated plus-maze (EPM) tests were performed in the septal OXTr neuron of hM3Dq $(n=26)$-, hM4Di $(n=13)-$, or mCherry $(n=19)$-transduced mice: (d) Time spent in the open arms of the EPM and (e) The number of entries in the open arms. Mice were treated with J60 i.p. 30 min before the OFT and EPM tests. Oneway ANOVA was used to analyze all panels. Mean \pm SEM; ${ }^{*} p<0.05 ;{ }^{* *} p<0.01 ;{ }^{* * *} p<0.001 ;{ }^{* * * *} p<0.0001$; ns not significant.

subpopulation of septum vGAT neurons. Accordingly, we stained septal OXTr neurons using anti-vGAT antibodies in brain sections of Oxtr-Cre mice transduced with hM3Dq-mCherry in the septal OXTr neurons. Consistently, we observed that most virally transduced OXTr neurons expressed vGAT, and approximately forty percent of vGAT-positive neurons expressed OXTr (Fig. 6a-d).
To further define the inhibitory projections of septal OXTr neurons to the HDB, we evaluated the HDB neuron action potential firing rates with optogenetic stimulation of OXTr neuron projections to the HDB in ChR2-transduced Oxtr-Cre mice (Fig. 6e). We performed loose-seal cell-attached recordings on HDB neurons in acute brain slices. Consistently, we observed that 
a

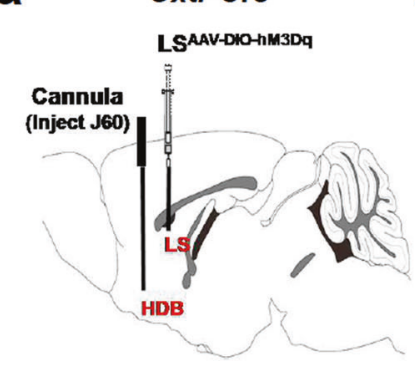

b

f

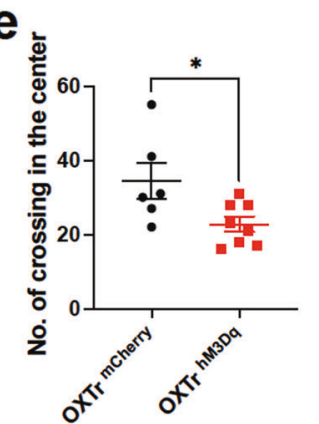

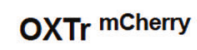

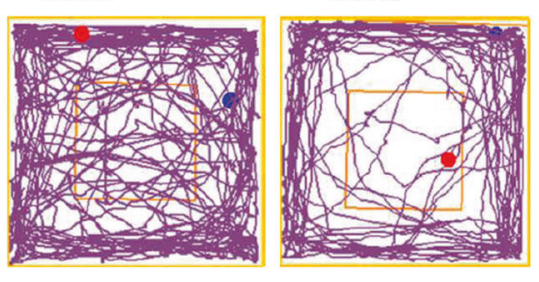

C

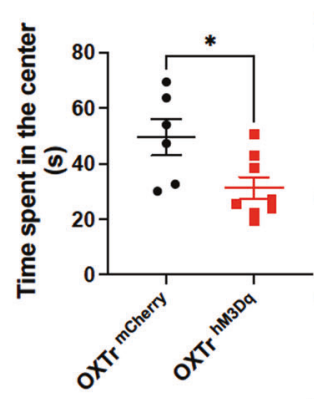

g
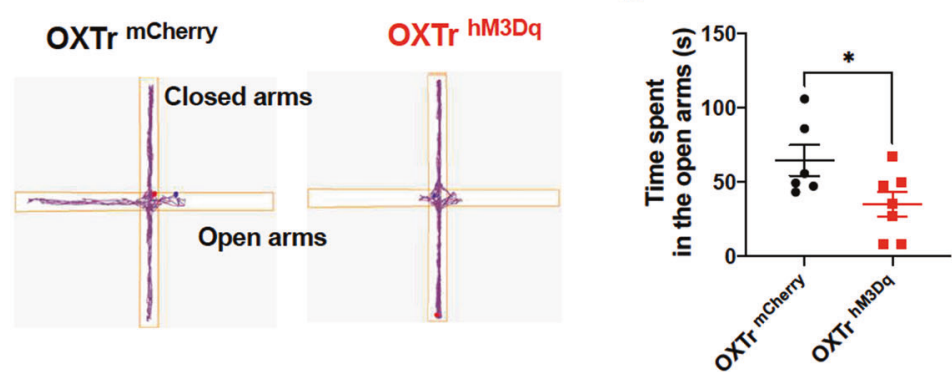

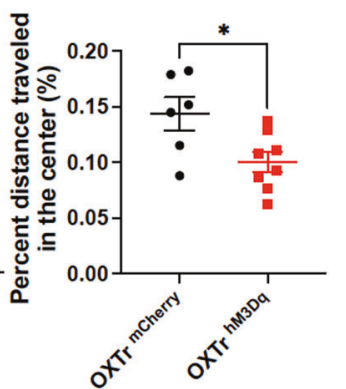

h

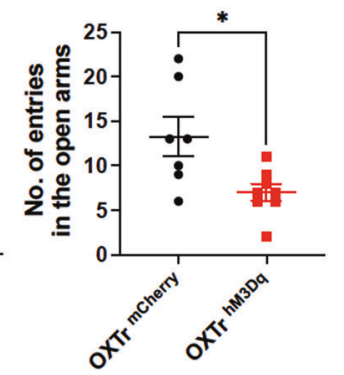

Fig. 4 Selective stimulation of septal OXTr neuron projections to HDB induced anxiety. a Schematic illustration of experimental design. A vector carrying Cre-dependent hM3Dq or mCherry was bilaterally targeted to the lateral septum, and a guide cannula was implanted for intraHDB injections in Oxtr-Cre mice. b Representative tracks of the open field test (OFT) in the septum of OXTr hM3Dq (right, $n=8$ )- and mCherry (left, $n=6$ )-transduced mice. c Time spent in the center of the open field. d Percent center distance traveled in the OFT. e The number of center entries in the OFT. $f$ Representative traces of the EPM recorded in the septal OXTr hM3Dq (right, $n=8)$ - and mCherry (left, $n=7$ )transduced mice. $\mathbf{g}$ Time spent in the open arms of the EPM. $\mathbf{h}$ The number of entries in the open arms. Two-tailed unpaired Student's $t$-tests were used to analyze all panels. Mean $\pm \mathrm{SEM} ;{ }^{*} p<0.05$; ns not significant.
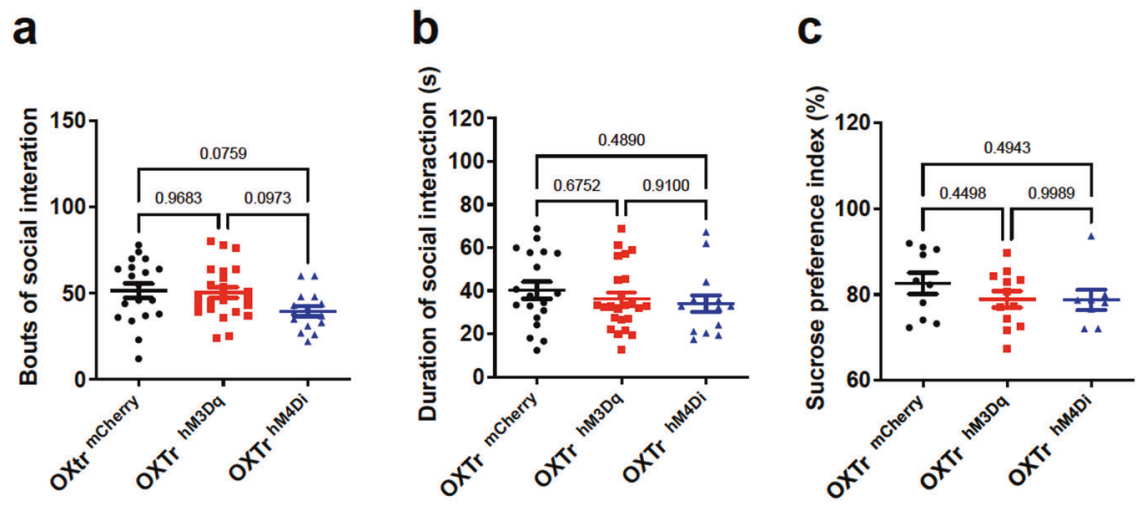

Fig. 5 Septal OXTr neurons had minimal effects on social interactions and depression-like behavior. No significant differences were detected in the septal OXTr neuron of hM3Dq $(n=24)$-, hM4Di $(n=15)$-, and mCherry $(n=19)$-transduced mice in social behavior and anhedonia tests. $\mathbf{a}-\mathbf{b}$ Social interaction test. c Sucrose preference test. Mean \pm SEM.

optogenetic stimulation of the ChR2-positive OXTr neuron projections to the HDB by shining blue light on the surface of the slices decreased the action potential firing rates in 8 of 15 recorded HDB neurons (Fig. $6 f-h$ ).

\section{The vHPC and its projections to the septum are activated under anxiety}

It remains unclear about the stimulatory drive to the septum under anxiogenic conditions. Our recent study shows that the vHPC [22], a well-established emotional brain region, project excitatory inputs to the septum. To define the activity of the vHPC under anxiety, we performed real-time wireless photometry monitoring of vHPC neuron activity in freely moving mice. We targeted a vector carrying the $\mathrm{Ca}^{2+}$ indicator $\mathrm{GCaMP}_{6 \mathrm{~s}}$ to the $\mathrm{vHPC}$ to transduce hippocampal neurons with the protein of $\mathrm{GCaMP}_{6 \mathrm{~s}}$ and implant a TeleFipho fiber-optic cannula over the virally targeted brain region (Fig. 7a).

After two weeks that allowed for $\mathrm{GCaMP}_{6 \mathrm{~s}}$ expression and one week of acclimation to carrying a headstage 15 min per day, mice were habituated to the behavioral test room $30 \mathrm{~min}$ before placing them in the light chamber of the LDB for 10 min with simultaneous real-time photometry monitoring of $\mathrm{GCaMP}_{6 \mathrm{~s}}$ signals. We observed that the average intensity of $\mathrm{GCaMP}_{6 \mathrm{~s}}$ signals recorded in the light chamber was more robust than in the dark chamber (Fig. 7b, c). Matching with the LDB results, we also evaluated vHPC neurons with the EPM, and $\mathrm{GCaMP}_{6 \mathrm{~s}}$ signals were increased when mice were exploring in the open arms compared to the closed arms (Fig. 7d). In cohort groups, we evaluated hippocampal projections to the 
a

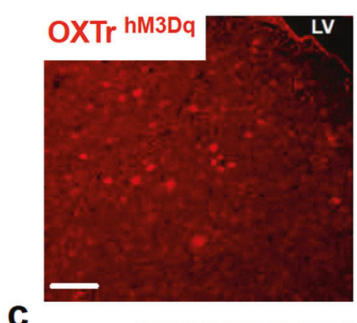

C

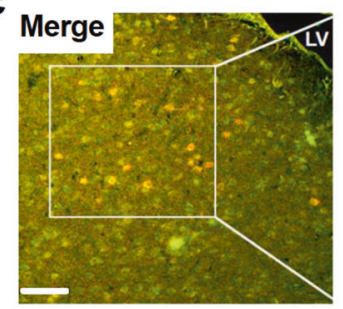

d

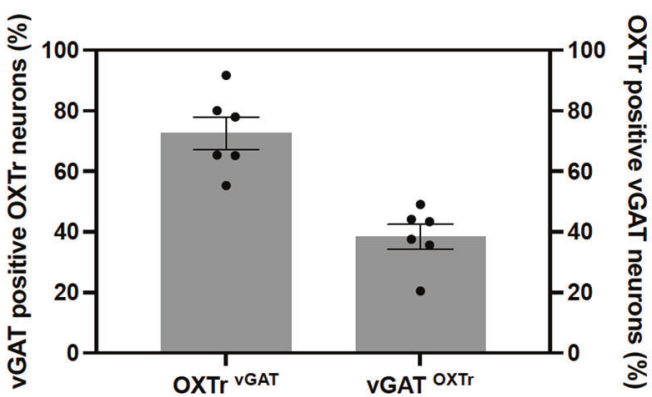

b
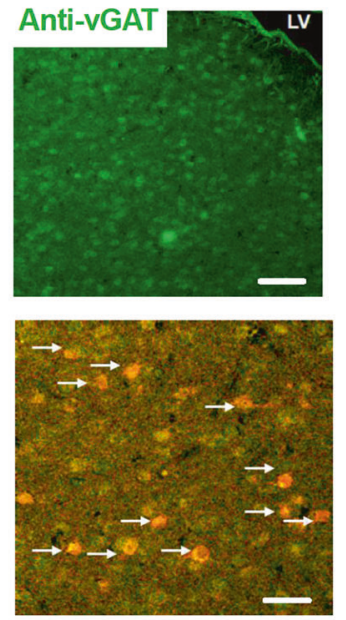

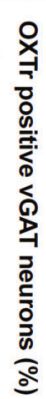

e
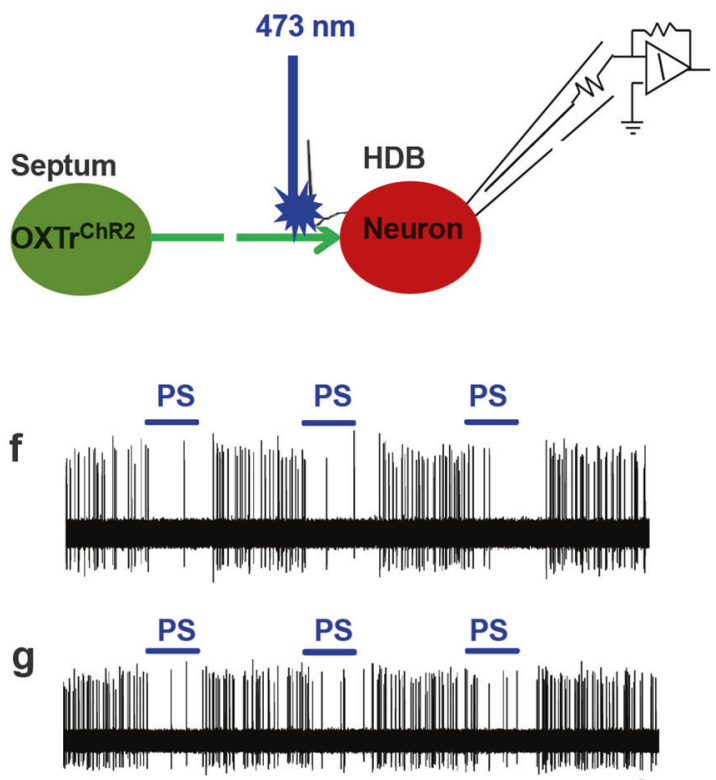

h

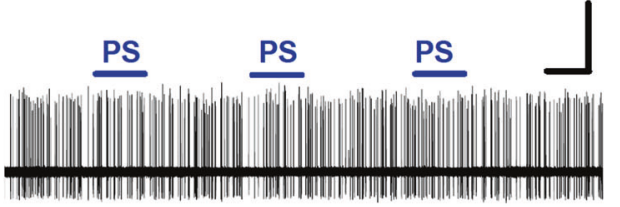

Fig. 6 Optogenetic stimulation of septal OXTr projections to the HDB inactivated HDB neurons. a-c Representative images of (a) hM3DqmCherry-transduced septal OXTr neurons, (b) anti-vGAT stains, and (c) overlap of hM3Dq-mCherry and vGAT. d Quantification of the percentage of vGAT-expressing OXTr and OXTr-expressing vGAT neurons ( $n=6$ per group). e A schematic illustration of loose-seal cellattached recordings at HDB neurons with optogenetic stimulation of ChR2-expressing OXTr projections to the HDB in acute brain slices of the OXTr neuron from ChR2-transduced Oxtr-Cre mice. f-h Optogenetic stimulation of septal OXTr neuron projections to the HDB decreased the action potential firing rates at eight of the 15 recorded HDB neurons: $(\mathbf{f}, \mathbf{g})$ two representative traces of PS-induced decrease in action potential firing rates recorded at two HDB neurons, and $(\mathbf{h})$ one representative trace that PS did not affect the firing rates. Mean \pm SEM. Scale bars, $50 \mu \mathrm{m}$ for $(\mathbf{a}, \mathbf{b}$, and $\mathbf{c}) ; 1 \mathrm{~s}$ and $1 \mathrm{mV}$ for $\mathrm{f}, \mathrm{g}$, and $\mathrm{h}$. PS photostimulation at $20 \mathrm{~Hz}$.

septum. We transduced vHPC neurons with a vector carrying an axon-targeted $\mathrm{Ca}^{2+}$ indicator $\mathrm{GCaMP}_{6 \mathrm{~s}}$ and implanted a TeleFipho cannula over the lateral septum (Fig. 7e). Consistently, we observed that the intensity of $\mathrm{GCaMP}_{6 \mathrm{~s}}$ signals recorded on hippocampal projections to the lateral septum was increased when mice were in the light chamber of the LDB (Fig. 7f, g) or the open arms of the EPM (Fig. 7h).

\section{DISCUSSION}

It is well-recognized that OXT elicits anxiolytic effects [34, 35] and promotes social reward [10-17] in rodents; however, human studies show that OXT administration increases recall of aversive events or stressful stimuli [19, 36, 37]. Furthermore, overexpression of OXTr in the septum exacerbates stress-induced fear [38]. These seemingly inconsistent findings might be attributable to different experimental paradigms and reconciled because OXTr is widely expressed in different brain regions. The functional roles of septal OXT signaling in anxiety and mood regulation remain to be defined, and the involved downstream targets await identification.

The septum nucleus is a limbic brain structure implicated in various cognitive and emotional processes [30, 39, 40]. Our recent studies show that chemo/optogenetic stimulation of ventral hippocampal glutamatergic inputs in the septum suppressed food intake [22], and activated septal vGAT and vGluT2 neurons reduced food intake by projecting to the lateral hypothalamus and paraventricular nucleus, respectively $[20,21]$. These results suggest that hippocampal suppression of feeding probably modulate hypothalamic feeding centers via the septum. This study observed that septal neurons expressing OXTr also expressed vGAT; however, it remains unclear whether and how these neurons participate in anxiety regulations. In this study, we found that chemogenetic activation of septal OXTr neurons induced anxiety-related behaviors. HDB probably mediated this anxiogenic effect as chemogenetic stimulation of septal OXTr neuron projections to the HDB elicited similar anxiogenic effects.

Meanwhile, optogenetic stimulation of the septal OXTr projections to the HDB inactivated the neurons localized within the HDB in our electrophysiology experiments. These results let us posit that septal OXTr neurons exerted anxiogenic effects probably by inhibiting HDB neurons via inhibitory GABAergic projections to the HDB. Therefore, our results suggest that the HDB neurons participated in the septal OXTr neuron anxiogenic effects; however, we cannot exclude other neural circuit pathways as septal neurons project to different brain regions.

The diagonal band of Broca (DB) is a basal forebrain region that consists of vertical (VDB) and HDB [41] limbs, projecting to the perilimbic, infralimbic, medial prefrontal cortices [42], hypothalamus [43], central nucleus of the amygdala and the septal nuclei [44]. Meanwhile, different neuronal populations, including cholinergic, GABAergic, and glutamatergic neurons, are intermingled in the HDB, and these neuronal populations project to different regions in mammalian brains [45-48]. It is also important to 


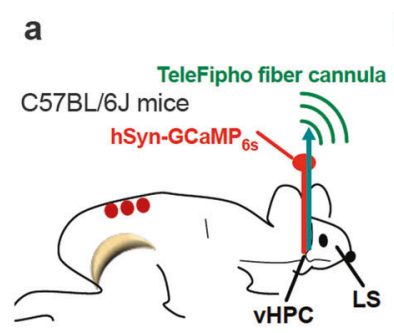

e

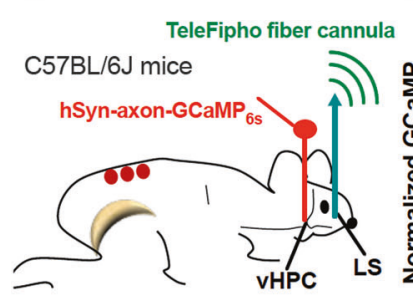

b

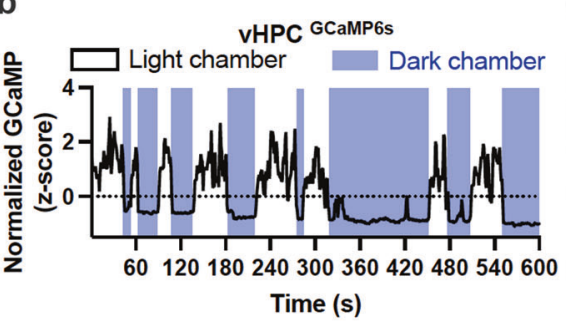

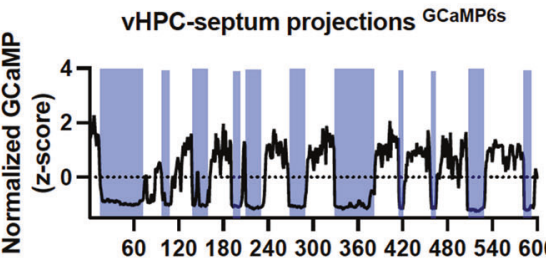

vHPC-septum projections GCaMP6s

Time (s)

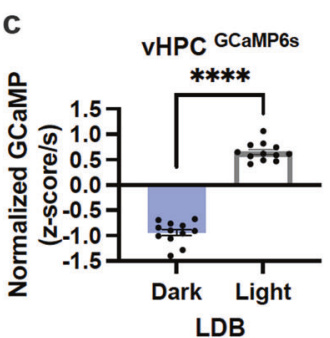

LDB

g

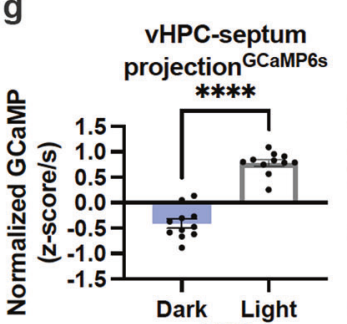

LDB

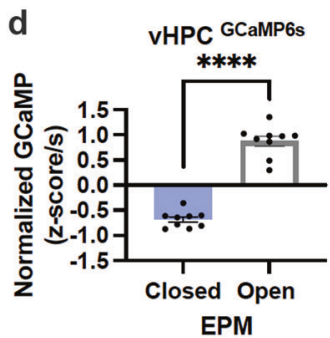

h

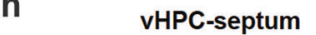
projection ${ }^{\text {GCaMP6s }}$

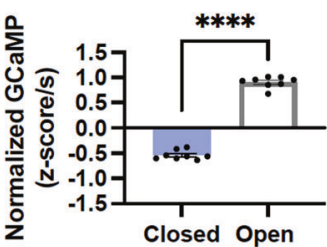

EPM

Fig. 7 The vHPC and its projections to the septum were excited under anxiogenic environments. a A schematic illustration of targeting a vector carrying $\mathrm{GCaMP}_{6 \mathrm{~s}}$ to the $\mathrm{vHPC}$ and implanting a wireless TeleFipho fiber cannula over the targeted site. b A representative trace of real-time wireless photometry monitoring of vHPC GCaMP 6 signals in light and dark chambers of the light-dark box (LDB). $\mathbf{c}$, $\mathbf{d}$ Group data of normalized $\mathrm{GCaMP}_{6 s}$ signals recorded in (c) LDB and (d) elevated plus-maze (EPM) tests. e An illustration of targeting a vector carrying axon-GCaMP 6 to the VHPC and implanting a wireless photometry fiber over the LS. $\mathbf{f} A$ representative trace of real-time monitoring of vHPC axon GCaMP 6 signals in the LS in the LDB. $\mathbf{g}, \mathbf{h}$ Group data of normalized vHPC axon GCaMP $6 \mathrm{~s}$ signals in the LS recorded in (g) LDB and (h) EPM. Each dot in $(\mathbf{c}, \mathbf{d}, \mathbf{g}, \mathbf{h})$ represents one animal. Mean \pm SEM; ${ }^{* * *} p<0.0001 ;$ vHPC ventral hippocampus, LS lateral septum.

mention that approximately 75 percent of septal OXTr neurons express vGAT and optogenetic stimulation of septal OXTr neuron projections to the HDB inhibited about 53 percent of HDB neurons, suggesting that $\mathrm{OXTr}$ neurons project to subpopulations of HDB neurons. Together, this septal and HDB neurochemical and anatomical heterogeneity determines the complexity of the neural circuits involved in the septal regulation of anxiety- and depressive-like behaviors, which are needed to be addressed in future studies. For example, a combined Cre-loxp and Flip-FRT system would be helpful to manipulate septal neurons and HDB cholinergic, glutamatergic and GABAergic neurons selectively.

There is emerging evidence indicating that cholinergic neurons send dense projections to the amygdala [46], a well-known brain region involved in anxiety regulation $[49,50]$, and cholinergic neurons express the inhibitory GABA neurotransmitters $[45,51]$. These results let us posit that septal OXTr neuron inhibition of GABA neurons in the HDB would induce anxiety by stimulating the amygdala via HDB disinhibition, which would participate in vHPC-dependent anxiety. However, we cannot exclude the involvement of other circuitries. Furthermore, our data also show that the VHPC and its projections to the septum are activated under anxiety, suggesting that the vHPC-projected neurons localized within the septum, including the OXTr neurons, and the subsequent descending septum to HDB to amygdala circuitries, probably partake in the VHPC-dependent anxiety, which awaits identification in the future.

It also is necessary and timely to point out that our data show that activation of septal OXTr neurons does not modulate depressive-like and prosocial behavior. However, it is wellrecognized that OXT actions promote multiple aspects of sociosexual behaviors and drug-seeking behavior. The complex OXT signaling in multiple mammalian brain regions and different experimental paradigms might explain these seemingly inconsistent results [1,52]. For example, earlier studies on the role of OXT in emotions have relied on systemic administration or knockdown, lacking cell-type specificity and spatiotemporal resolution. Our study avoided these limitations as we combined chemogenetics and Cre-transgenic animals, allowing us to manipulate neuron activities in a spatiotemporal manner selectively. These differential findings suggest that the neural circuitry for the septal regulation of anxiety and mood might be distinct or partially overlapping. Meanwhile, we observed that inactivation of septal OXTr neurons elicited minimal effects on anxiety- and depressive-like behaviors, which may imply that these neurons are silent in baseline conditions.

Increased locomotion may be a confounding factor in behavioral tests such as the forced swim test (FST) [53-55]. However, studies using a drug or an animal model of depression support that changes in immobility in the FST may occur independently of changes in other behaviors [53,56,57]. In our studies, locomotor activity change was significant but small and unlikely to impact the other behavioral tests. Other factors may also influence behavioral tests, such as pre-exposure to tail suspension test (TST) or other stressors [58]. Thus, if a drug or procedure is shown to modulate anxiety and locomotion, these effects do not necessarily need to be related.

Collectively, we report functional roles of septal OXT signaling in the regulation of anxiety and identified the involved downstream target, which might serve as a target for therapeutic interventions in minimizing unintended side effects of OXT.

\section{REFERENCES}

1. Jurek B, Neumann ID. The oxytocin receptor: from intracellular signaling to behavior. Physiol Rev 2018;98:180-1908.

2. Dabrowska J, Hazra R, Ahern TH, Guo JD, McDonald AJ, Mascagni F, et al. Neuroanatomical evidence for reciprocal regulation of the corticotrophin-releasing factor and oxytocin systems in the hypothalamus and the bed nucleus of the stria terminalis of the rat: implications for balancing stress and affect. Psychoneuroendocrinology 2011;36:1312-26.

3. Mittaud P, Labourdette G, Zingg H, Guenot-Di Scala D. Neurons modulate oxytocin receptor expression in rat cultured astrocytes: involvement of TGF-beta and membrane components. Glia 2002;37:169-77.

4. Hidema S, Fukuda T, Hiraoka Y, Mizukami H, Hayashi R, Otsuka A, et al. Generation of Oxtr cDNA (HA)-Ires-Cre mice for gene expression in an oxytocin receptorspecific manner. J Cell Bio 2016;117:1099-111. 
5. Li K, Nakajima M, Ibanez-Tallon I, Heintz N. A cortical circuit for sexually dimorphic oxytocin- dependent anxiety behaviors. Cell 2016;167:60-72.

6. Yoshida M, Takatanagi Y, Inoue K, Kimura T, Young LJ, Onaka T, et al. Evidence that oxytocin exerts anxiolytic effects via oxytocin receptor expressed in serotonergic neurons in mice. J Neurosci 2009;29:2259-71.

7. Grinevich V, Desarmenien MG, Chini B, Tauber M, Muscatelli F. Ontogenesis of oxytocin pathways in the mammalian brain: late maturation and psychosocial disorders. Front Neuroanat 2014;8:164.

8. Zimmerman EA, Nilaver G, Hou-Yu A, Silverman AJ. Vasopressinergic and oxytocinergic pathways in the central nervous system. Fed Proc 1984;43:91-96.

9. Gimpl G, Fahrenholz F. The oxytocin receptor system: structure, function, and regulation. Physiol Rev 2001;81:629-83.

10. Dolen G, Darvishzadeh A, Huang KW, Malenka R. Social reward requires coordinated activity of nucleus accumbens oxytocin and serotonin. Nature 2013;501:179-84.

11. Shamay-Tsoory SG, Abu-Akel A. The social salience hypothesis of oxytocin. Biol Psychiatry 2016;79:194-202.

12. Hung LW, Neuner S, Polepalli JS, Beier KT, Wright M, Walsh JJ, et al. Gating of social reward by oxytocin in the ventral tegmental area. Science 2017;357:1406-11.

13. Meyer Lindenberg A, Domes G, Kirsch P, Heinriches MA. Oxytocin and vasopressin in the human brain: social neuropeptides for translational medicine. Nat Rev Neurosci 2011;12:524-38.

14. De Dreu CKW, Greer LL, Handgraaf MJJ, Shalvi S, Van Kleef GA, Baas M, et al. The neuropeptide oxytocin regulates altruism in intergroup conflict among humans. Science 2010:328:1408-11.

15. Declerk $\mathrm{CH}$, Boone $\mathrm{C}$, Kiyonari T. Oxytocin and cooperation under conditions of uncertainty: the modulating role of incentives and social information. Horm Behav 2010;57:368-74.

16. Viviani $D$, Charlet $A$, van den Burg E, Robinet $C$, Hurni N, Abatis $M$, et al. Oxytocin selectively gates fear responses through distinct outputs from the central amygdala. Science 2011;333:104-7.

17. Neumann ID, Slattery DA. Oxytocin in general anxiety and social fear: a translational approach. Biol Psychiatry 2015;79:213-21.

18. Matsuzaki $M$, Matsushita $H$, Tomizawa $K$, Matsui $H$. Oxytocin: a therapeutic target for mental disorders. J Physiol Sci 2012;62:441-4.

19. Grillon C, Krimsky M, Charney DR, Vytal K, Ernst M, Cornwell B. Oxytocin increases anxiety to unpredictable threat. Mol Psychiatry 2012;18:958-60.

20. Sweeney $P$, Yang Y. An inhibitory septum to lateral hypothalamus circuit that suppresses feeding. J Neurosci 2016;36:11185-95.

21. Sweeney P, Li C, Yang Y. Appetite suppressive role of medial septal glutamatergic neurons. Proc Natl Acad Sci Usa 2017:114:13816-21.

22. Sweeney $P$, Yang Y. An excitatory ventral hippocampus to lateral septum circuit that suppresses feeding. Nat Commun 2015;6:10188.

23. Li C, Hou Y, Zhang J, Sui G, Du X, Licinio J, et al. AGRP neurons modulate fastinginduced anxiolytic effects. Transl Psychiatry 2019;9:111.

24. Zhang J, Chen D, Sweeney P, Yang Y. An excitatory ventromedial hypothalamus (VMH) to paraventricular thalamus (PVT) circuit that suppresses food intake. Nat Commun 2020;11:6326.

25. Sweeney P, Qi Y, Xu Z, Yang Y. Activation of hypothalamic astrocytes suppresses feeding without altering emotional states. Glia 2016;64:2263-73.

26. Sweeney $P$, Levack R, Watters J, Xu Z, Yang Y. Caffeine increases food intake while reducing anxiety-related behaviors. Appetite 2016;101:171-7.

27. Liu MY, Yin CY, Zhu LJ, Zhu XH, Xu C, Luo CX, et al. Sucrose preference test for measurement of stress-induced anhedonia in mice. Nat Protoc 2018;13:1686-98.

28. Selimbeyoglu A, Kim CK, Inoue M, Lee SY, Hong ASO, Kauvar I, et al. Modulation of prefrontal cortex excitation/inhibition balance rescues social behavior in CNTNAP2-deficient mice. Sci Transl Med 2017;9:eaah6733.

29. Penagarikano O, Abrahams BS, Herman El, Winden KD, Gdalyahu A, Dong $H$, et al. Absence of CNTNAP2 leads to epilepsy, neuronal migration abnormalities, and core autism-related deficits. Cell 2011;147:235-46.

30. Menon R, Grund T, Zoicas I, Althammer F, Fiedler D, Biermeier V, et al. Oxytocin signaling in the lateral septum prevents social fear during lactation. Curr Biol 2018;28:1066-78.

31. Sheehan TP, Chambers RA, Russell DS. Regulation of affect by the lateral septum: implications for neuropsychiatry. Brain Res Brain Res Rev 2004;46:71-117.

32. McIntire SL, Reimer RJ, Schuske K, Edwards RH, Jorgensen EM. Identification and characterization of the vesicular GABA transporter. Nature 1997;389:870-6.

33. Chaudhry FA, Reimer RJ, Bellocchio EE, Danbolt NC, Osen KK, Edwards RH, et al. The vesicular GABA transporter, vGAT, localized to synaptic vesicles in sets of glycinergic as well as GABAergic neurons. J Neurosci 1998;18:9733-50.

34. Carter CS. Neuroendocrine perspectives on social attachment and love. Psychoneuroendocrinology 1998;23:779-818.
35. Ayers LW, Missig G, Schulkin J, Rosen JB. Oxytocin reduces background anxiety in a fear-potentiated startle paradigm: peripheral vs. central administration. Neuropsychopharmacology 2011;36:2488-97.

36. Striepens N, Scheele D, Kendrick KM, Becker B, Schafer L, Schwalba K, et al. Oxytocin facilitates protective responses to aversive social stimuli in males. Proc Natl Acad Sci Usa 2012;109:18144-9.

37. Bartz JA, Zaki J, Ochsner KN, Bolger N, Kolevzon A, Ludwig N, et al. Effects of oxytocin on recollections of maternal care and closeness. Proc Natl Acad Sci Usa 2010;107:21371-5.

38. Guzman YF, Tronson NC, Jovasevic V, Sato K, Guedea AL, Mizukami H, et al. Fearenhancing effects of septal oxytocin receptors. Nat Neurosci 2013;16:1185-9.

39. Bakshi VP, Newman SM, Smith-Roe S, Jochman KA, Kalin NH. Stimulation of lateral septum CRF2 receptors promotes anorexia and stress-like behaviors: functional homology to CRF1 receptors in basolateral amygdala. J Neurosci 2007;27:10568-77.

40. Singewald GM, Rjabokon A, Singewald N, Ebner K. The modulatory role of the lateral septum on neuroendocrine and behavioral stress responses. Neuropsychopharmacology 2011;36:793-804.

41. Nasimi A, Hatam M. GABA and glutamate receptors in the horizontal limb of diagonal band of Broca (HDB): effects on cardiovascular regulation. Exp Brain Res 2005;167:268-75.

42. Gaykema RP, Luiten PG, Nyakas C, Traber J. Cortical projection patterns of the medial septum-diagonal band complex. J Comp Neurol 1990;293:103-24.

43. Jhamandas JH, Raby W, Rogers J, Buijs RM, Renaud LP. Diagonal band projection towards the hypothalamic supraoptic nucleus: light and electron microscopic observations in the rat. J Comp Neurol 1989;282:15-23.

44. Tomimoto H, Kamo H, Kameyama M, McGeer PL, Kimura H. Descending projections of the basal forebrain in the rat demonstrated by the anterograde neural tracer Phaseolus vulgaris leucoagglutinin (PHA-L). Brain Res 1987;425:248-55.

45. Case DT, Burton SD, Gedeon JY, Williams SPG, Urban NN, Seal RP. Layer- and cell type- selective co-transmission by a basal forebrain cholinergic projection to the olfactory bulb. Nat Commun 2017;8:652.

46. Agostinelli LJ, Geerling JC, Scammell TE. Basal forebrain subcortical projections. Brain Struct Funct 2019;224:1097-117.

47. Yang C, McKenna JT, Brown RE. Basal forebrain glutamatergic and GABAergic neurons: intrinsic properties and modulation by cholinergic inputs and hypnotic agents. Sleep 2015;39:A0075.

48. Zaborszky L, Van den Pol A, Gyengesi E The basal forebrain cholinergic projection system in mice. In: Watson C, Paxinos G, Puelles $L$, editors. The mouse nervous system. London: Elsevier. 2012;P.684-718.

49. Lee SC, Amir A, Haufler D, Pare D. Differential recruitment of competing valencerelated amygdala networks during anxiety. Neuron 2017;96:81-88.

50. Prager EM, Bergstrom HC, Wynn GH, Braga MF. The basolateral amygdala gamma- aminobutyric acidergic system in health and disease. J Neurosci Res 2016:94:548-67.

51. Saunders A, Granger AJ, Sabatini BL. Corelease of acetylcholine and GABA from cholinergic forebrain neurons. Elife 2015;4:e06412.

52. Quintana DS, Rokicki J, van der Meer D, Alnas D, Kaufmann T, Cordova-Palomera A, et al. Oxytocin pathway gene networks in the human brain. Nat Commun 2019:10:1038.

53. Hilakivi LA, Lister RG. Correlations between behavior of mice in Porsolt's swim test and in tests of anxiety, locomotion, and exploration. Behav Neural Biol 1990;53:153-9.

54. Thornton EW, Bradbury GE, Davies C. Increased immobility in an automated forced swimming test following lesion of the habenula in rats: absence of evidence for a contribution from motor impairment. Behav Neurosci 1990;104:37-43.

55. Bogdanova OV, Kanekar S, D'Anci KE, Renshaw PF. Factors influencing behavior in the forced swim test. Physiol Behav 2013;118:227-39.

56. Borsini F, Meli A. Is the forced swimming test a suitable model for revealing antidepressant activity? Psychopharmacology 1988;94:147-60.

57. Porsolt RD, Pichon MLE, Jalfre M. Depression: a new animal model sensitive to antidepressant treatments. Nature 1977;266:730-2.

58. Kale PP, Addepalli V, Ghadawale SR. Impact of pre-exposure of tail suspension on behavioral parameters like locomotion, exploration, and anxiety in mice. Indian J Exp Biol 2013;51:732-8.

\section{ACKNOWLEDGEMENTS}

This study was supported by the NIH (R01 MH109441; R01 DK112759, to YY) and Einstein Research Foundation. We thank Guangzhi Sui for performing OXTr mice 
genotyping, viral injections, and cannula implantation for some experiments. We also thank Dr. Jia Zhang for helping setting up and testing the TeleFipho system. We thank all the members of the Yang laboratory for discussing and providing critical comments to this study, and thank the scientists who deposited the relevant vectors used in this study.

\section{AUTHOR CONTRIBUTIONS}

YY conceived and designed the study; TH performed behavioral tests; YY performed electrophysiology and photometry experiments; TH and $Y Y$ analyzed the data and plotted the figures; YY, MLW, and TH wrote, and JL, and FG edited the manuscript.

\section{COMPETING INTERESTS}

The authors declare no competing interests.

\section{ADDITIONAL INFORMATION}

Correspondence and requests for materials should be addressed to Yunlei Yang.
Reprints and permission information is available at http://www.nature.com/reprints

Publisher's note Springer Nature remains neutral with regard to jurisdictional claims in published maps and institutional affiliations.

(i) Open Access This article is licensed under a Creative Commons Attribution 4.0 International License, which permits use, sharing, adaptation, distribution and reproduction in any medium or format, as long as you give appropriate credit to the original author(s) and the source, provide a link to the Creative Commons license, and indicate if changes were made. The images or other third party material in this article are included in the article's Creative Commons license, unless indicated otherwise in a credit line to the material. If material is not included in the article's Creative Commons license and your intended use is not permitted by statutory regulation or exceeds the permitted use, you will need to obtain permission directly from the copyright holder. To view a copy of this license, visit http://creativecommons. org/licenses/by/4.0/.

(c) The Author(s) 2021 